\title{
CDISC SDTM Musculoskeletal System Finding Test Name Terminology
}

National Cancer Institute

\section{Source}

National Cancer Institute. CDISC SDTM Musculoskeletal System Finding Test Name

Terminology. NCI Thesaurus. Code C127270.

Terminology associated with the musculoskeletal system finding test name codelist of the Clinical Data Interchange Standards Consortium (CDISC) Study Data Tabulation Model (SDTM). 